\title{
DISTANCE $k$-DOMINATION IN SOME CYCLE RELATED GRAPHS
}

\author{
S. K. VAIDYA AND N. J. KOTHARI
}

Received 12 March, 2014

\begin{abstract}
In this paper we determine distance $k$-domination number of graph obtained by duplication of vertices altogether by edges in cycle $C_{n}$, splitting graph of cycle $C_{n}$ as well as graph obtained by duplication of edges altogether by vertices in cycle $C_{n}$.
\end{abstract}

2010 Mathematics Subject Classification: 05C69; 05C12; 05C38; 05 C76

Keywords: dominating set, domination number, distance domination, splitting graph

\section{INTRODUCTION}

Graph $G=(V(G), E(G))$, we mean simple, finite, connected and undirected graph. The open neighbourhood $N(v)$ of $v \in V(G)$ is the set of all vertices adjacent to $v$. That is, $N(v)=\{u \in V(G) / u v \in E(G)\}$. The closed neighbourhood of $v \in V(G)$ is the set $N[v]=N(v) \cup\{v\}$. The distance $d(u, v)$ between two vertices $u$ and $v$ is the length of the shortest $u v$-path in $G$, if exists, otherwise $d(u, v)=\infty$. The open $k$-neighbourhood $N_{k}(v)$ of a vertex $v \in V(G)$ is the set of all vertices of $G$ which are different from $v$ and at a distance at most $k$ from $v$ in $G$. That is $N_{k}(v)=\{u \in V(G) / d(u, v) \leq k\}$. The closed $k$-neighbourhood set is defined as $N_{k}[v]=N_{k}(v) \cup\{v\}$. It is obvious that $N(v)=N_{1}(v)$. A set $D \subseteq V(G)$ is called a dominating set if every vertex in $V(G)-D$ is adjacent to at least one vertex in $D$. For terminology and notation not defined here we follow West [13] and Haynes et al. [3]. The concept of distance dominating set was initiated by Slater [7] with special reference to communication network, while the term distance $k$-dominating set was given by Henning et al. [5]. For an integer $k \geq 1, D \subseteq V(G)$ is a distance $k$-dominating set of $G$, if every vertex in $V(G)-D$ is within the distance $k$ from some vertex $v \in D$. That is, $N_{k}[D]=V(G)$. The minimum cardinality among all the distance $k$-dominating sets of $G$ is called the distance $k$-domination number of $G$ and it is denoted by $\gamma_{k}(G)$. It is obvious that $\gamma(G)=\gamma_{1}(G)$. A distance $k$-dominating set of cardinality $\gamma_{k}(G)$ is called a $\gamma_{k}-$ set. Many reserchers have explored the concept of distance $k$-domination in graphs. The distance domination number for cartesian products of two paths has been investigated by Klobucar [6] 
while the distance domination in the context of spanning tree of the graph is discussed by Griggs and Hutchinson [2]. The bounds on the distance two-domination number and the classes of graphs attaining these bounds are reported in Sridharan et al. [8]. Tian and $\mathrm{Xu}$ [9] have established upper bound for distance $k$-domination for connected graph $G$ and show that $\gamma_{k}(G) \leq\left\lfloor\frac{n-\Delta+k-1}{k}\right\rfloor$. The same authors in [10] have studied average distance and distance domination number and established an upper bound of average distance in terms of distance domination. Fischermann and Volkmann [1] have characterized the graphs whose distance $n$-domination number is equal to half of their number of vertices, when the diameter is greater or equal to $2 n-1$. Vaidya and Kothari [12] have investigated distance $k$-domination number of total graph, shadow graph and middle graph of path $P_{n}$. The same authors in [11] have investigated distance $k$-domination number for the graphs obtained by graph operations on some standard graphs. For more bibliographic references on distance $k$-domination, the readers are advised to refer a survey article by Henning [4].

\section{Results}

Proposition 1 ([4]). Let $k \geq 1$ and $D$ be a distance $k$-dominating set of a graph $G$. Then $D$ is a minimal distance $k$-dominating set of $G$ if and only if each $d \in D$ has at least one of the following two properties hold.

(1) There exist a vertex $v \in V(G)-D$ such that $N_{k}(v) \cap D=\{d\}$.

(2) The vertex $d$ is at distance at least $k+1$ from every other vertex $d$ of $D$ in $G$.

Definition 1. Duplication of a vertex $v$ by a new edge $e=v^{\prime} v^{\prime \prime}$ of graph $G$ produces a new graph $G^{\prime}$ such that $N\left(v^{\prime}\right) \cap N\left(v^{\prime \prime}\right)=\{v\}$.

Theorem 1. If $G$ is a graph obtained by duplication of vertices altogether by edges in cycle $C_{n}(n \leq 2 k-1)$ then $\gamma_{k}(G)=1$.

Proof. Let $G$ be a graph obtained by duplication of vertices $v_{1}, v_{2}, \ldots, v_{n}$ by edges $u_{2 i-1} u_{2 i}(1 \leq i \leq n)$ in cycle $C_{n}$. Then $\left.D=\left\{v_{\lfloor} \mid \frac{n}{2}\right\rfloor\right\}$ is distance $k$-dominating set of $G$ as $n \leq 2 k-1$. Hence $\gamma_{k}(G)=1$.

Theorem 2. If $G$ is a graph obtained by duplication of vertices altogether by edges in cycle $C_{n}(n>2 k-1)$ then

$$
\gamma_{k}(G)= \begin{cases}\left\lfloor\frac{n}{2 k-1}\right\rfloor+1, & \text { for } n \equiv 1,2, \ldots, 2 k-2(\bmod 2 k-1) \\ \frac{n}{2 k-1} & \text { for } n \equiv 0(\bmod 2 k-1)\end{cases}
$$

Proof. Let $G$ be a graph obtained by duplication of vertices $v_{1}, v_{2}, \ldots, v_{n}$ by edges $u_{2 i-1} u_{2 i}(1 \leq i \leq n)$ in cycle $C_{n}$. One can observe that $v_{i}$ 's dominate more vertices than $u_{i}$ 's. Here vertices from $v_{n-(k-1)}$ to $v_{k+1}, u_{2 n-(2 k-3)}$ to $u_{2 n}$ and $u_{1}$ 
to $u_{2 k}$ are dominated by a vertex $v_{1}$ at a distance $k$. Also $d\left(v_{1}, u_{2 k}\right)=k$, and $d\left(u_{2 n-(2 k-3)}, v_{1}\right)=k$. Hence $2 k-1$ consecutive vertices from $u_{i}$ 's dominated by only one vertex $v_{1}$. Hence

$$
\gamma_{k}(G) \geq\left\lfloor\frac{n}{2 k-1}\right\rfloor
$$

Now depending upon the number of vertices of $C_{n}$, consider the following subsets, For $n \equiv 1,2, \ldots, 2 k-2(\bmod 2 k-1)$

$$
D=\left\{v_{1+(2 k-1) j} / 0 \leq j \leq\left\lfloor\frac{n}{2 k-1}\right\rfloor\right\},|D|=\left\lfloor\frac{n}{2 k-1}\right\rfloor+1,
$$

for $n \equiv 0(\bmod 2 k-1)$

$$
D=\left\{v_{1+(2 k-1) j} / 0 \leq j<\frac{n}{2 k-1}\right\},|D|=\frac{n}{2 k-1} .
$$

We claim that each $D$ is a distance $k$-dominating set as For $j \neq 0$,

$$
\begin{aligned}
& \qquad d\left(v_{1+(2 k-1) j}, v_{i+(2 k-1) j}\right) \leq k, \text { where }-k+1 \leq i \leq k+1, i \neq 1 \\
& d\left(v_{1+(2 k-1) j}, u_{i+(2 k-1) j}\right) \leq k, \text { where }(3-2 k)+(2 k-1) j \leq i \leq 2 k+(2 k-1) j \\
& \text { and for } j=0,
\end{aligned}
$$

$$
\begin{gathered}
d\left(v_{1}, v_{i}\right) \leq k, \text { where } n-k+1 \leq i \leq n, 2 \leq i \leq k+1 \\
d\left(v_{1}, u_{i}\right) \leq k, \text { where } 1 \leq i \leq 2 k, 2 n-(2 k-3) \leq i \leq 2 n
\end{gathered}
$$

Therefore for $j \neq 0$,

$$
\begin{aligned}
N_{k}\left(v_{1+(2 k-1) j}\right)=\{ & v_{2+(2 k-1) j}, v_{3+(2 k-1) j}, \ldots, v_{(k+1)+(2 k-1) j}, \ldots, v_{(2 k-1) j}, \\
& v_{(2 k-1) j-1}, \ldots, v_{(2 k-1) j-(k-1)}, u_{1+2(2 k-1) j}, u_{2(1+(2 k-1) j)}, \\
& \ldots, u_{2 k+2((2 k-1) j)}, u_{(3-2 k)+2((2 k-1) j)}, u_{(4-2 k)+2((2 k-1) j)}, \\
& \left.\ldots, u_{2(2 k-1) j}\right\} .
\end{aligned}
$$

While for $j=0$,

$$
\begin{aligned}
N_{k}\left(v_{1}\right)=\{ & v_{2}, v_{3}, \ldots, v_{k+1}, v_{n-(k-1)}, v_{n-k}, \ldots, v_{n}, u_{1}, u_{2}, \ldots, u_{2 k}, u_{2 n}, \\
& \left.u_{2 n-1}, \ldots, u_{2 n-(2 k-3)}\right\} .
\end{aligned}
$$

Then $N_{k}[D]=N_{k}\left[v_{1}\right] \cup N_{k}\left[v_{1+(2 k-1) j}\right]=V(G)$.

For some $j=j_{1}, v_{1+(2 k-1) j_{1}} \in D$ and for some $j=j_{2}, v_{1+(2 k-1) j_{2}} \in D$,

$$
d\left(v_{1+(2 k-1) j_{1}}, v_{1+(2 k-1) j_{2}}\right)=\left(j_{2}-j_{1}\right)(2 k-1) \geq k+1 .
$$

Which implies that every vertex $d$ of $D$ is at a distance $k+1$ apart from every other vertex of $D$ in $G$. Thus by Proposition 1 above defined $D$ is a minimal distance 
$k$-dominating set of $G$ and by expression 2.1 it is also of minimum cardinality for $n>2 k-1$. Hence

$$
\gamma_{k}(G)= \begin{cases}\left\lfloor\frac{n}{2 k-1}\right\rfloor+1, & \text { for } n \equiv 1,2, \ldots, 2 k-2(\bmod 2 k-1) \\ \frac{n}{2 k-1} & \text { for } n \equiv 0(\bmod 2 k-1)\end{cases}
$$

Illustration 1. Distance 3-dominating set in graph $G$ obtained by duplication of vertices in cycle $C_{22}$ altogether by edges is shown by solid vertices in FIGURE 1.

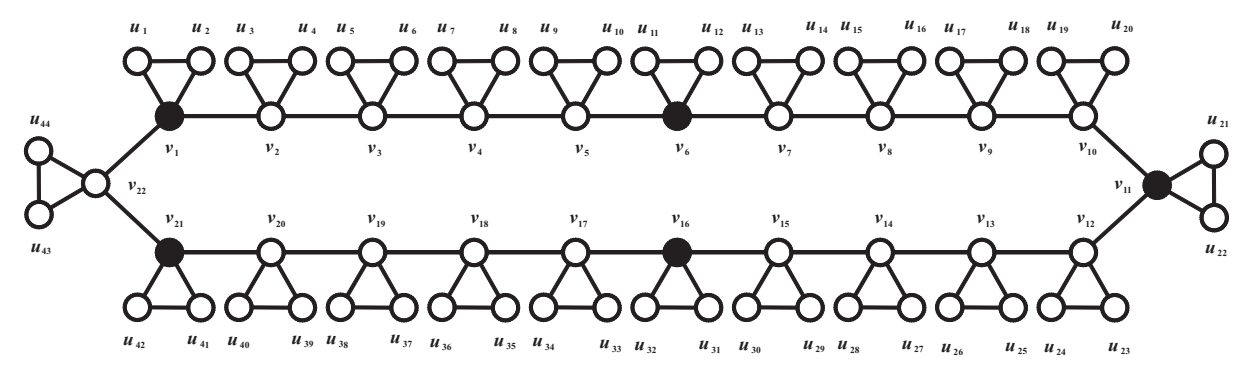

FIGURE 1.

Definition 2. For a graph $G$, the splitting graph $S^{\prime}(G)$ of graph $G$ is obtained by adding a new vertex $v^{\prime}$ corresponding to each vertex $v$ of $G$ such that $N(v)=N\left(v^{\prime}\right)$.

Theorem 3. If $n \leq 2 k+1, k \neq 1$, then $\gamma_{k}\left(S^{\prime}\left(C_{n}\right)\right)=1$.

Proof. Let $v_{1}, v_{2}, \ldots, v_{n}$ be the vertices of cycle $C_{n}$ and $u_{1}, u_{2}, \ldots, u_{n}$ be the vertices corresponding to $v_{1}, v_{2}, \ldots, v_{n}$ which are added to obtain $S^{\prime}\left(C_{n}\right)$. Then $D=$ $\left\{v_{\lceil}\left\lceil\frac{n}{2}\right\rceil\right\}$ is distance $k$-dominating set of $S^{\prime}\left(C_{n}\right)$ as $n \leq 2 k+1$. Hence $\gamma_{k}\left(S^{\prime}\left(C_{n}\right)\right)=$ 1 .

Theorem 4. If $n>2 k+1$ then

$$
\gamma_{k}\left(S^{\prime}\left(C_{n}\right)\right)= \begin{cases}\left\lfloor\frac{n}{2 k+1}\right\rfloor+1, & \text { for } n \equiv 1,2, \ldots, 2 k(\bmod 2 k+1) \\ \frac{n}{2 k+1} & \text { for } n \equiv 0(\bmod 2 k+1)\end{cases}
$$

Proof. Let $v_{1}, v_{2}, \ldots, v_{n}$ be the vertices of cycle $C_{n}$ and $u_{1}, u_{2}, \ldots, u_{n}$ be the vertices corresponding to $v_{1}, v_{2}, \ldots, v_{n}$ which are added to obtain $S^{\prime}\left(C_{n}\right)$. One can observe that $v_{i}$ 's dominate more vertices than $u_{i}$ 's at a distance $k$. In graph $S^{\prime}\left(C_{n}\right)$ 
vertices $v_{n-(k-1)}$ to $v_{k+1}$ and $u_{n-(k-1)}$ to $u_{n+k}$ are dominated by a vertex $v_{1}$ at a distance $k$. Also $d\left(v_{n-(k-1)}, v_{k+1}\right)=2 k+1$, and $d\left(u_{n-(k-1)}, u_{k+1}\right)=2 k+1$. Hence $2 k+1$ consecutive vertices from $v_{i}$ 's and $(2 k+1)$ consecutive vertices from $u_{i}$ 's dominated by only one vertex at a distance $k$. Which implies that

$$
\gamma_{k}\left(S^{\prime}\left(C_{n}\right)\right) \geq\left\lfloor\frac{n}{2 k+1}\right\rfloor
$$

Now depending upon the number of vertices of $C_{n}$, consider the following subsets, For $n \equiv 1,2, \ldots, 2 k(\bmod 2 k+1)$

$$
D=\left\{v_{1+(2 k+1) j} / 0 \leq j \leq\left\lfloor\frac{n}{2 k+1}\right\rfloor\right\},|D|=\left\lfloor\frac{n}{2 k+1}\right\rfloor+1,
$$

for $n \equiv 0(\bmod 2 k+1)$

$$
D=\left\{v_{1+(2 k+1) j} / 0 \leq j<\frac{n}{2 k+1}\right\},|D|=\frac{n}{2 k+1} .
$$

We claim that each $D$ is a distance $k$-dominating set as for $j \neq 0$,

$$
\begin{gathered}
d\left(v_{1+(2 k+1) j}, v_{i+(2 k+1) j}\right) \leq k, \text { where }-k+1 \leq i \leq k+1, i \neq 1 \\
d\left(v_{1+(2 k+1) j}, u_{i+(2 k+1) j}\right) \leq k, \text { where }-k+1 \leq i \leq k+1
\end{gathered}
$$

while for $j=0$

$$
\begin{aligned}
& d\left(v_{1}, v_{i}\right) \leq k, \text { where } n-k+1 \leq i \leq n, 1 \leq i \leq k+1 \\
& d\left(v_{1}, u_{i}\right) \leq k, \text { where } 1 \leq i \leq k+1, n-k+1 \leq i \leq n
\end{aligned}
$$

Therefore $j \neq 0$,

$$
\begin{aligned}
N_{k}\left(v_{1+(2 k+1) j}\right)=\{ & v_{2+(2 k+1) j}, v_{3+(2 k+1) j}, \ldots, v_{(k+1)+(2 k+1) j}, v_{(2 k+1) j}, \\
& v_{(2 k+1) j-1}, \ldots, v_{(2 k+1) j-(k-1)}, u_{1+(2 k+1) j}, u_{2+(2 k+1) j}, \ldots, \\
& \left.u_{(k+1)+(2 k+1) j}, u_{(2 k+1) j}, u_{(2 k+1) j-1}, \ldots, u_{(2 k+1) j-(k-1)}\right\} .
\end{aligned}
$$

While for $j=0$,

$$
\begin{aligned}
N_{k}\left(v_{1}\right)= & \left\{v_{2}, v_{3}, \ldots, v_{k+1}, v_{n}, v_{n-1}, v_{n-(-k+1)}, u_{1}, u_{2}, \ldots, u_{k+1}, u_{n-k+1},\right. \\
& \left.u_{n-k+2}, \ldots, u_{n}\right\} .
\end{aligned}
$$

Then $N_{k}[D]=N_{k}\left[v_{1}\right] \cup N_{k}\left[v_{1+(2 k+1) j}\right]=V\left(S^{\prime}\left(C_{n}\right)\right)$.

For some $j=j_{1}, v_{1+(2 k+1) j_{1}} \in D$ and for some $j=j_{2}, v_{1+(2 k+1) j_{2}} \in D$,

$$
d\left(v_{1+(2 k+1) j_{1}}, v_{1+(2 k+1) j_{2}}\right)=\left(j_{2}-j_{1}\right)(2 k+1) \geq k+1
$$

This implies that every vertex $d$ of $D$ is at a distance $k+1$ apart from every other vertex of $D$ in $S^{\prime}\left(C_{n}\right)$. Thus by Proposition 1 above defined $D$ is a minimal distance 
$k$-dominating set of $G$ and by expression 2.2 it is also of minimum cardinality for $n>2 k-1$. Hence

$$
\gamma_{k}\left(S^{\prime}\left(C_{n}\right)\right)= \begin{cases}\left\lfloor\frac{n}{2 k+1}\right\rfloor+1, & \text { for } n \equiv 1,2, \ldots, 2 k(\bmod 2 k+1) \\ \frac{n}{2 k+1} & \text { for } n \equiv 0(\bmod 2 k+1)\end{cases}
$$

Illustration 2. Distance 4-dominating set in $S^{\prime}\left(C_{20}\right)$ is shown by solid vertices in FIGURE 2.

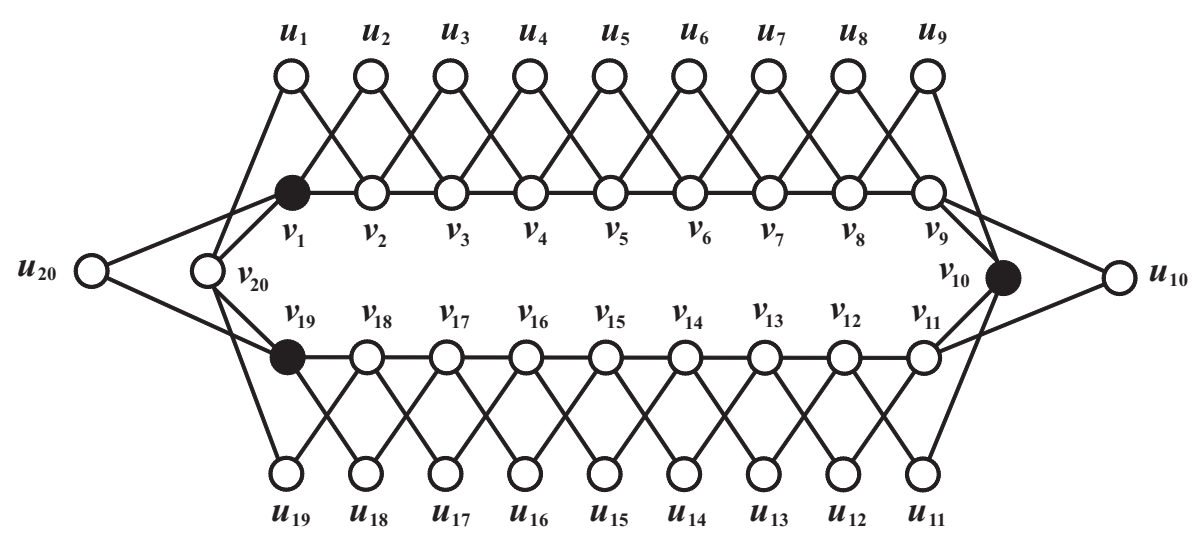

FIGURE 2.

Definition 3. Duplication of an edge $e=u v$ by a new vertex $w$ in a graph $G$ produces a new graph $G^{\prime}$ such that $N(w)=\{u, v\}$.

Theorem 5. If $G$ is a graph obtained by duplication of edges altogether by vertices in cycle $C_{n}$ $(n \leq 2 k)$ then $\gamma_{k}(G)=1$.

Proof. Let $G$ be a graph obtained by duplication of edges $v_{i} v_{i+1}$ altogether by vertices $u_{i},(1 \leq i<n)$ in cycle $C_{n}$. Then $\left\{v_{\left\lfloor\frac{n}{2}\right\rfloor}\right\rfloor$ is distance $k$-dominating set of $G$ as $n \leq 2 k$. Hence $\gamma_{k}(G)=1$. 
Theorem 6. If $G$ is a graph obtained by duplication of edges altogether by vertices in cycle $C_{n}(n>2 k)$ then

$$
\gamma_{k}(G)= \begin{cases}\left\lfloor\frac{n}{2 k}\right\rfloor+1, & \text { for } n \equiv 1,2, \ldots, 2 k-1(\bmod 2 k) \\ \frac{n}{2 k} & \text { for } n \equiv 0(\bmod 2 k)\end{cases}
$$

Proof. Let $\mathrm{G}$ be a graph obtained by duplication of edges $v_{i} v_{i+1}$ altogether by vertices $u_{i}(1 \leq i<n)$ in cycle $C_{n}$. One can observe that $v_{i}$ 's dominate more vertices than $u_{i}$ 's at a distance $k$. In graph $G$ vertices $v_{n-k}$ to $v_{k+1}$ and $u_{n-(k-1)}$ to $u_{k}$ are dominated by a vertex $v_{1}$ at a distance $k$. Also $d\left(u_{n-(k-1)}, v_{1}\right)=k$ and $d\left(v_{1}, u_{k}\right)=$ $k$. Hence $2 k$ consecutive vertices of $u_{i}$ 's are dominated by only one vertex at a distance $k$. Hence

$$
\gamma_{k}(G) \geq\left\lfloor\frac{n}{2 k}\right\rfloor
$$

Now depending upon the number of vertices of $C_{n}$, consider the following subsets.

For $n \equiv 1,2, \ldots, 2 k-1(\bmod 2 k)$

$$
D=\left\{v_{1+(2 k) j} / 0 \leq j \leq\left\lfloor\frac{n}{2 k}\right\rfloor\right\},|D|=\left\lfloor\frac{n}{2 k}\right\rfloor+1,
$$

for $n \equiv 0(\bmod 2 k)$

$$
D=\left\{v_{1+(2 k) j} / 0 \leq j<\frac{n}{2 k}\right\},|D|=\frac{n}{2 k} .
$$

Now we claim that each $D$ is a distance $k$-dominating set as for $j \neq 0$,

$$
\begin{gathered}
d\left(v_{1+2 k j}, v_{i+2 k j}\right) \leq k, \text { where }-k+1 \leq i \leq k+1, i \neq 1, \\
d\left(v_{1+2 k j}, u_{i+2 k j}\right) \leq k, \text { where }-k+1 \leq i \leq k
\end{gathered}
$$

while for $j=0$,

$$
\begin{gathered}
d\left(v_{1}, v_{i}\right) \leq k, \text { where } n-k+1 \leq i \leq n, 2 \leq i \leq k+1 \\
d\left(v_{1}, u_{i}\right) \leq k, \text { where } n-k+1 \leq i \leq n, 1 \leq i \leq k
\end{gathered}
$$

Therefore for $j \neq 0$,

$$
\begin{aligned}
N_{k}\left(v_{1+2 k j}\right)=\{ & v_{2+2 k j}, v_{3+2 k j}, \ldots, v_{(k+1)+2 k j}, v_{2 k j}, v_{2 k j-1}, \ldots, v_{2 k j-(k-1)}, \\
& \left.u_{1+2 k j}, u_{2+2 k j}, \ldots, u_{k+2 k j}, u_{2 k j}, u_{2 k j-1}, \ldots, u_{2 k j-(k-1)}\right\} .
\end{aligned}
$$

While for $j=0$,

$$
\begin{aligned}
N_{k}\left(v_{1}\right)= & \left\{v_{2}, v_{3}, \ldots, v_{k+1}, v_{n-k+1}, v_{n-k+2}, \ldots, v_{n}, u_{1}, u_{2}, \ldots, u_{k}, u_{n-k+1},\right. \\
& \left.u_{n-k+2}, \ldots, u_{n}\right\} .
\end{aligned}
$$


This implies that $N_{k}[D]=N_{k}\left[v_{1}\right] \cup N_{k}\left[v_{1+(2 k) j}\right]=V(G)$.

For some $j=j_{1}, v_{1+(2 k) j_{1}} \in D$ and for some $j=j_{2}, v_{1+(2 k) j_{2}} \in D$,

$$
d\left(v_{1+(2 k) j_{1}}, v_{1+(2 k) j_{2}}\right)=\left(j_{2}-j_{1}\right) 2 k \geq k+1
$$

Which implies that every vertex $d$ of $D$ is at a distance $k+1$ apart from every other vertex of $D$ in $G$. Thus by Proposition 1 above defined $D$ is a minimal distance $k$-dominating set of $G$ and by expression 2.3 it is also of minimum cardinality for $n>2 k$. Hence

$$
\gamma_{k}(G)= \begin{cases}\left\lfloor\frac{n}{2 k}\right\rfloor+1, & \text { for } n \equiv 1,2, \ldots, 2 k-1(\bmod 2 k) \\ \frac{n}{2 k} & \text { for } n \equiv 0(\bmod 2 k)\end{cases}
$$

Illustration 3. Distance 2-dominating set in graph $G$ obtained by duplication of edges in $C_{18}$ altogether by vertices is shown by solid vertices in FIGURE 3.

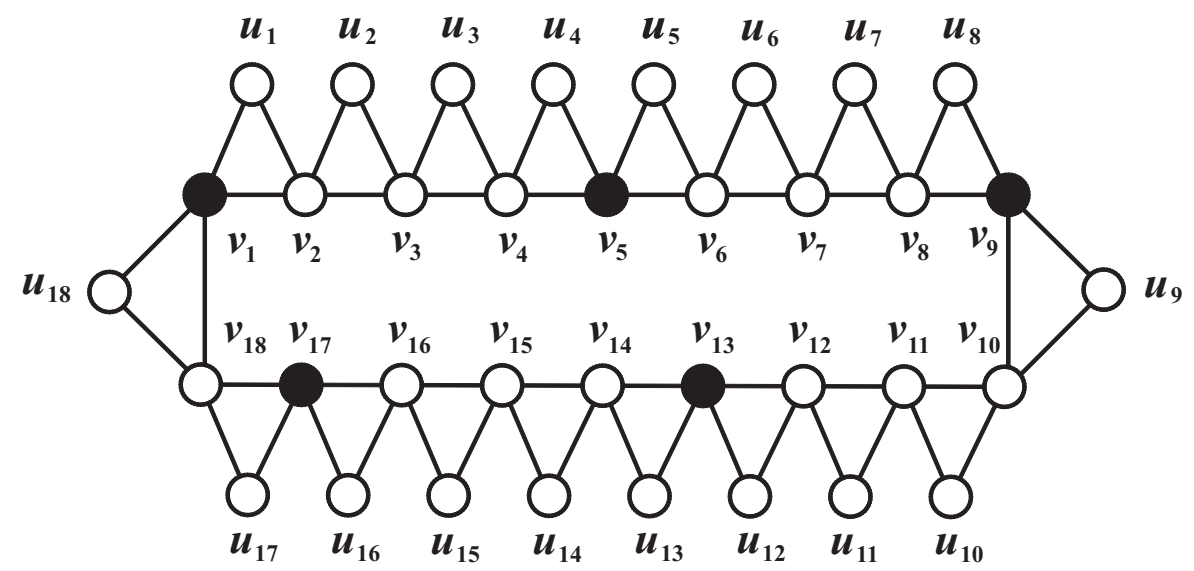

FIGURE 3.

\section{REFERENCES}

[1] M. Fischermann and L. Volkmann, "Graphs having distance- $n$ domination number half their order." Discrete Appl. Math., vol. 120, no. 1-3, pp. 97-107, 2002, doi: 10.1016/S0166218X(01)00284-0.

[2] J. R. Griggs and J. P. Hutchinson, "On the $r$-domination number of a graph.” Discrete Math., vol. 101, no. 1-3, pp. 65-72, 1992, doi: 10.1016/0012-365X(92)90591-3.

[3] T. W. Haynes, S. T. Hedetniemi, and P. J. Slater, Fundamentals of Domination in Graphs. Marcel Dekker, New York, 1998. 
[4] M. A. Henning, "Distance domination in graphs," in Domination in Graphs: Advanced Topics, T. W. Haynes, S. T. Hedetniemi, and P. J. Slater, Eds. Marcel Dekker, New York, 1998, pp. 321-349.

[5] M. Henning, O. R. Oellermann, and H. C. Swart, "Bounds on distance domination parameters." J. Comb. Inf. Syst. Sci., vol. 16, no. 1, pp. 11-18, 1991.

[6] A. Klobučar, "On the $k$-dominating number of Cartesian products of two paths." Math. Slovaca, vol. 55, no. 2, pp. 141-154, 2005.

[7] P. J. Slater, "R-domination in graphs." J. Assoc. Comput. Mach., vol. 23, pp. 446-450, 1976, doi: $10.1145 / 321958.321964$.

[8] N. Sridharan, V. Subramanian, and M. Elias, "Bounds on the distance two-domination number of a graph." Graphs Comb., vol. 18, no. 3, pp. 667-675, 2002, doi: 10.1007/s003730200050.

[9] F. Tian and J.-M. Xu, "A note on distance domination numbers of graphs." Australas. J. Comb., vol. 43, pp. 181-190, 2009.

[10] F. Tian and J.-M. Xu, "Average distances and distance domination numbers." Discrete Appl. Math., vol. 157, no. 5, pp. 1113-1127, 2009, doi: 10.1016/j.dam.2008.03.024.

[11] S. K. Vaidya and N. J. Kothari, "Distance k-domination of some path related graphs," International Journal of Mathematics and Soft Computing, vol. 4, no. 1, pp. 1-5, 2014.

[12] S. K. Vaidya and N. J. Kothari, "Some new results on distance $k$-domination in graphs." Int. J. Comb., vol. 2013, p. 4, 2013, doi: 10.1155/2013/795401.

[13] D. B. West, Introduction to graph theory, 2nd ed. Prentice-Hall, New Delhi, India, 2003.

Authors' addresses

S. K. Vaidya

Saurashtra University, Department of Mathematics, Rajkot - 360005, Gujarat, INDIA

E-mail address: samirkvaidya@yahoo.co.in

N. J. Kothari

L. E. College (Diploma), General Department, Morbi-363642, Gujarat, INDIA

E-mail address: nirang_kothari@yahoo.com 